\title{
A Rare Case of Idiopathic Pyometra in a Premenopausal Patient
}

\author{
Kristina M. Mori ${ }^{1}$, Howard D. Epstein ${ }^{2}$, Michael C. Roossin ${ }^{3}$, Bram H. Goldstein ${ }^{4}$ \\ ${ }^{1}$ Gynecologic Oncology Associates, Newport Beach, CA, USA, ${ }^{2}$ Department of Pathology, Hoag Hospital, Newport Beach, CA, USA, \\ ${ }^{3}$ Department of Radiology, Hoag Hospital, Newport Beach, CA, USA, ${ }^{4}$ The Women's Cancer Research Foundation, Newport Beach, CA, USA
}

Pyometra is a very uncommon disease principally occurring in postmenopausal women. It is characterized by the accumulation of purulent material within the uterine cavity. This paper presents the clinical history of a 35-year-old premenopausal woman with otherwise normal menstruation who developed heavy menstruation and was diagnosed with a benign pyometra of indeterminate etiology in March 2017. The patient underwent repeated ultrasound-guided drainage, dilation and curettage, and antibiotic therapy. Biopsies of the pelvic sidewall revealed endometriosis in June 2017. The heavy menstruation and suppurative fluid in the uterus of the patient persisted in which intramuscular leuprolide acetate was prescribed to address the endometriosis and heavy menstrual bleeding. Ultimately, the leuprolide acetate effectively resolved the patient's bleeding and pyometra. The medication was concluded after 12 months of supervision and the patient is currently symptom free. Pyometra is an unusual condition rarely identified in premenopausal women. Drainage and antibiotic therapy are routinely employed; however, one may consider gonadotropin-releasing hormone agonist medication to potentially confer a beneficial patient outcome in rare cases where endometriosis and bleeding are intractable.

Key Words: Endometriosis, GnRH agonists, Hemorrhaging, Premenopausal status, Pyometra

\section{INTRODUCTION}

Pyometra is an unusual disease that is characterized by the formation and accretion of purulent material within the uterus, and frequently attributed to an impaired or obstructed, evacuative process [1]. The reported incidence of pyometra in gynecologic patients is $0.01 \%-0.05 \%$ and chiefly afflicts postmenopausal women with concomitant morbidities [2].

Pyometra can also develop in response to a cervical or uterine malignancy [3] and is potentially a consequence of prior radiotherapy $[1,4]$. Alternatively, benign indications, namely leiomyoma, endometrial polyps, postoperative cervical occlusion and structural anomalies (e.g., imperforate hymen), have been implicated in the development of this disease. Clinically, a pyometra often coincides with suppurative discharge, postmeno- pausal bleeding, and lower abdominal pain [4].

Idiopathic pyometra has been rarely documented [5], especially in premenopausal patients $[2,6]$. Moreover, recurrent disease following ultrasound-guided suction is uncommon [2]. In the current study, we recount a difficult case involving a premenopausal woman with a chronic pyometra who was successfully managed with leuprolide acetate.

\section{CASE REPORT}

A 35-year-old, nulligravid woman with a history of pheochromocytoma, Cushing's disease, polycystic ovarian syndrome, hypothyroidism and otherwise regular menstruation, presented to our gynecologic oncology service with endometrial hemorrhaging and loculated collections of purulent material within the uterus in

Received: July 9, 2020 Revised: September 16, 2020 Accepted: October 19, 2020

Address for Correspondence: Bram H, Goldstein, The Women's Cancer Research Foundation, 351 Hospital Road, Suite 506, Newport Beach, CA 92663, USA

Tel: 1-949-642-5165, E-mail: bramhg1969@gmail.com, ORCID: https://orcid.org/0000-0002-9827-2105

Copyright (C) by The Korean Society of Menopause

(c) This is an Open Access article distributed under the terms of the Creative Commons Attribution Non-Commercial License (http://creativecommons.org/licenses/by-nc/4.0/). 
March 2017. The patient underwent a diagnostic laparoscopy, dilation and curettage with ultrasound-guided suction to address the nearly $3 \mathrm{~L}$ of fluid encountered in her pelvis. The fluid was submitted for cytologic evaluation, which revealed benign endometrial cells. Thereafter, the results from a right pelvic sidewall biopsy revealed polypoid endometrium with focal hyperplasia and no atypia.

In September 2017, the patient developed dark, vaginal hemorrhaging and passage of tissue. She was evaluated via ultrasound, which exhibited a $4.8 \mathrm{~cm}$ complex cyst and approximately $500 \mathrm{~mL}$ of malodorous fluid and tissue. There was no evidence of cervical stenosis or discernible structural damage.

The patient underwent ultrasound-guided suction, dilation and curettage, and bilateral robotic-assisted pelvic sidewall biopsies to preclude a malignant process; pathology revealed endometriosis with abundant fibrin and inflammatory exudate (Fig. 1). The cultures grew out Peptostreptococcus and following infectious disease consultation, the patient began ampicillin (500 $\mathrm{mg}$ ) and amoxicillin (875 mg) therapy; additionally, she was treated with tranexamic acid $(650 \mathrm{mg})$ and medroxyprogesterone $(10 \mathrm{mg})$ to manage her heavy menstrual bleeding.

In October 2017, the patient complained of pelvic pain and a putrid, brown discharge, whereupon she was admitted to the emergency room for further evaluation. An ultrasound revealed a distended uterus, measuring $16.3 \mathrm{~cm} \times 14.6 \mathrm{~cm} \times 8.9 \mathrm{~cm}$, with considerable heterogeneous fluid in the endometrium (Fig. 2). Dur-

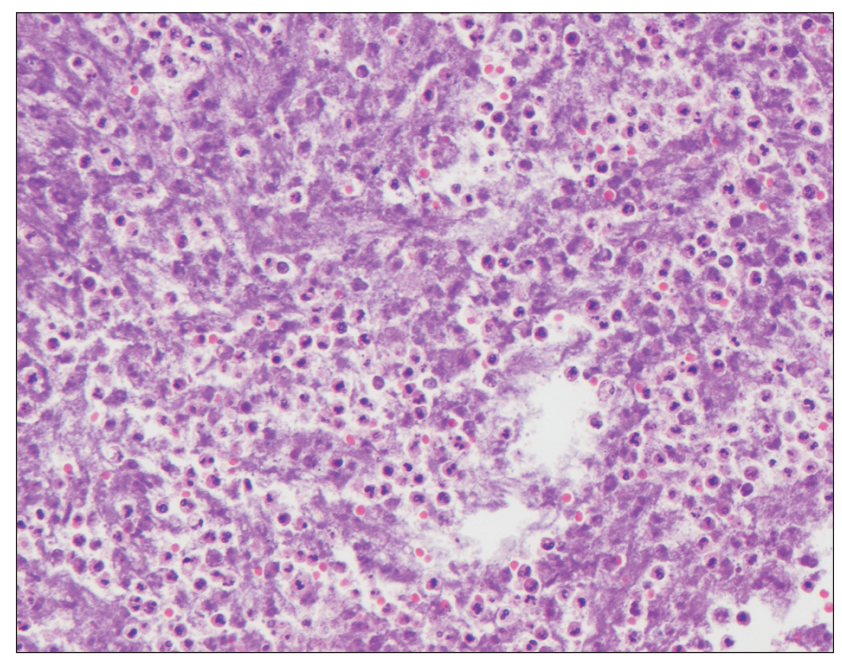

Fig. 1. Tissue from endometrial curettage depicting inflammatory exudate (H\&E, $\times 200 ; 20 \times$ objective and $10 \times$ ocular). ing laparoscopic examination, the patient underwent dilation and curettage with ultrasound guidance to ensure complete evacuation of the endometrial lining. The fluid was submitted for pathologic evaluation, of which the cultures grew out Streptococcus anginosus; the patient was treated with ceftriaxone $(500 \mathrm{mg})$, metronidazole $(500 \mathrm{mg})$, and amoxicillin $(625 \mathrm{mg})$. A subsequent computed tomography angiography of the pelvis excluded an arteriovenous malformation but detected significant accumulation of fluid.

In November 2017, the patient had an ultrasoundguided suction with dilation and curettage; cultures of Citrobacter freundii were isolated and identified. The patient was administered cefdinir $(300 \mathrm{mg})$ and metronidazole $(500 \mathrm{mg})$; due to the persistence of the pyometra, the ultrasound-guided suction with dilation and curettage was repeated in December 2017 (Fig. 3) and the patient was then treated with intramuscular methylergonovine maleate and carboprost tromethamine.

In March 2018, the patient complained of pelvic pain and was spontaneously admitted to the emergency room; intraoperatively, a 1,200 $\mathrm{mL}$ hemoperitoneum was identified and subsequently evacuated. Another pelvic sidewall biopsy was performed, which revealed endometriosis, fibrosis and tubal metaplasia. Subsequently, the patient commenced with leuprolide acetate (11.25 mg, intramuscular) every 3 months, which effectively addressed the endometriosis and persistent heavy menstrual bleeding. The patient concluded her

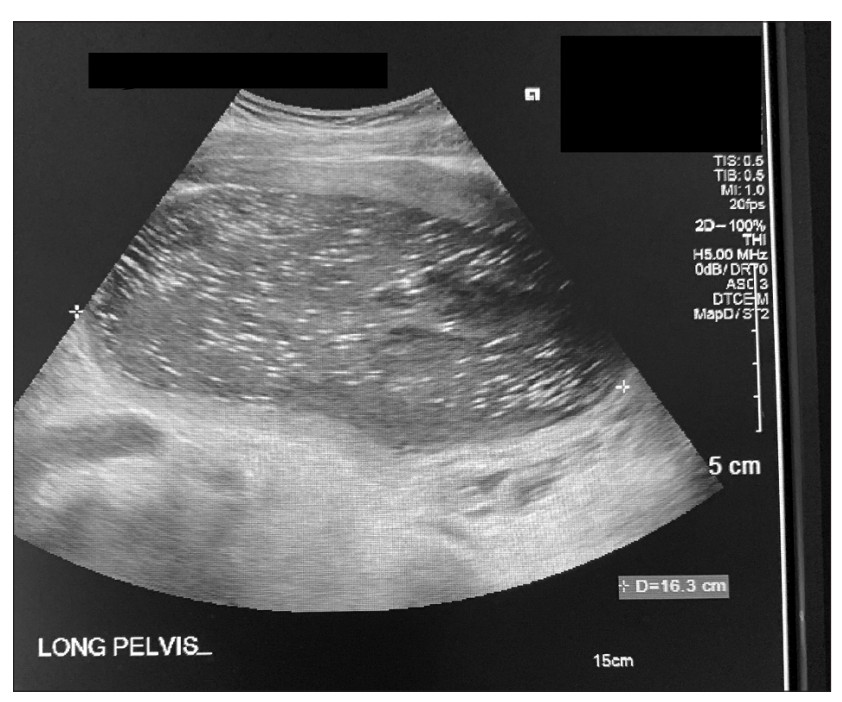

Fig. 2. October 2017 pelvic ultrasound image depicting a large amount of complex fluid in the endometrial cavity, resulting in marked distension; also, gas is present within the endometrial cavity. 




Fig. 3. Edematous fragment of endometrial tissue with acute and chronic inflammation (H\&E, $\times 200 ; 20 \times$ objective and $10 \times$ ocular).

leuprolide acetate regimen after 12 months and is currently not taking further medication to treat the pyometra; she remains symptom free and in accordance with a February 2019 ultrasound (Fig. 4), the pyometra was ostensibly resolved.

\section{DISCUSSION}

Pyometra is caused by a natural drainage impairment within the cervix, often attributed to a structural process (e.g., cervical stenosis), malignant pathology or secondary to radiotherapy [1,3,7]. Additionally, urogenital anomalies, namely atrophic cervicitis, have reportedly contributed to the disease's manifestation $[2,6,8]$.

Characteristically, the symptoms of pyometra include postmenopausal bleeding, purulent, vaginal discharge and uterine enlargement although they can also be relatively nonspecific [1]. Moreover, the diagnosis of pyometra can be further elusive in asymptomatic patients, especially when they are premenopausal; the median age at onset for a pyometra is 65 years with an incidence of $13.6 \%$ in elderly patients $[2,9]$.

In the current study, we report on a very unusual case of idiopathic, benign pyometra involving a premenopausal woman. Despite repeated ultrasound-guided evacuation, the purulent fluid continued to manifest itself; also, the vaginal hemorrhaging continued. Multiple streptococcal species were identified and two successive pelvic sidewall biopsies corroborated endometriosis. Ultimately, the patient commenced with 12 months of

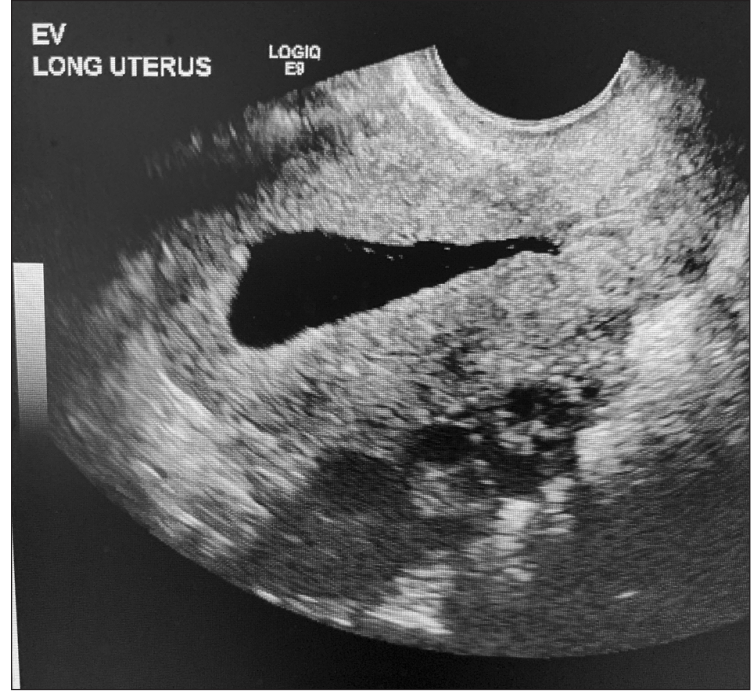

Fig. 4. February 2019 pelvic ultrasound image exhibiting marked improvement in the pyometrium.

leuprolide acetate to manage the endometriosis and persistent bleeding, which also secondarily addressed the pyometra.

Remarkably, there was neither an underlying malignancy nor any structural deficit to account for the pyometra. Alternatively, the patient's history was significant for numerous co-morbidities (e.g., pheochromocytoma, Cushing's disease) and while we never diagnosed a malignant indication, one may conjecture that the persistent nature of her condition was secondary to the multiple procedures she underwent.

In female patients, leuprolide acetate reduces the amount of estrogen produced; as such, $\mathrm{GnRH}$ analogs are utilized to treat endometriosis and temporarily control excessive uterine bleeding [10]. Consequently, since a pyometra can result from hormonal and structural changes in the uterine lining, potentially treating endometriosis with leuprolide acetate in a patient with a history of Cushing's disease was also resultingly effective at resolving the infectious process [11,12].

Interestingly, following a review of the literature, we ascertained that long-term-release $\mathrm{GnRH}$ agonists were effective at treating pyometra in canines [13] although we were unable to identify any reported clinical studies. However, one gynecologic case documented a patient with hidradenitis suppurativa of the vulva and perineum who was successfully managed with antibiotics, dexamethasone and leuprolide acetate [14].

Pyometra should be initially considered secondary to a gynecological malignancy until determined 
otherwise. Moreover, despite the propensity of this condition to afflict postmenopausal women, our case illustrates the significance of including premenopausal women when considering the differential diagnosis. Ultrasound has significant clinical utility and Doppler imaging is also beneficial, particularly in evaluating blood flow changes associated with an intercurrent endometrial cancer [15]. In persistent cases of pyometra, repeat drainage is indicated; conversely, if the condition is intractable, definitive surgical management is recommended, particularly when conservative measures have been exhausted or an underlying malignancy cannot be excluded [2]. Finally, routine surveillance is warranted to promptly detect or avert substantial hemorrhaging, uterine rupture and peritonitis, all of which may significantly increase the incidence of patient morbidity and mortality [9].

\section{ACKNOWLEDGMENTS}

This study was supported by the Women's Cancer Research Foundation and Joan and Len Rullo, in memory of Elizabeth Johnson.

\section{CONFLICT OF INTEREST}

No potential conflict of interest relevant to this article was reported.

\section{REFERENCES}

1. Muram D, Drouin P, Thompson FE, Oxorn H. Pyometra. Can Med Assoc J 1981; 125: 589-92.

2. Chan LY, Lau TK, Wong SF, Yuen PM. Pyometra. What is its clinical significance? J Reprod Med 2001; 46: 952-6.

3. Kerimoglu OS, Pekin A, Yilmaz SA, Bakbak BB, Celik C. Pyome- tra in elderly post-menopausal women: a sign of malignity. Eur J Gynaecol Oncol 2015; 36: 59-61.

4. Imachi M, Tanaka S, Ishikawa S, Matsuo K. Spontaneous perforation of pyometra presenting as generalized peritonitis in a patient with cervical cancer. Gynecol Oncol 1993; 50: 384-8.

5. Akazawa K, Takamori H, Yasuda H. [Clinico-statistical study on pyometra in high-aged outpatients]. Nihon Sanka Fujinka Gakkai Zasshi 1991; 43: 1539-45. Japanese.

6. Batioglu S, Zeyneloglu HB. Endoscopic management of a case of complete septate uterus with unilateral pyometra. Gynecol Obstet Invest 1999; 47: 144-6.

7. Carter B, Jones CP, Ross RA, Thomas WL. A bacteriologic and clinical study of pyometra. Am J Obstet Gynecol 1951;62:793-805.

8. Lui MW, Cheung VY, Pun TC. Clinical significance of pyometra. J Reprod Med 2015; 60: 329-32.

9. Chaopotong P, Benjapibal M, Thamkhantho M. Spontaneous perforation of pyometra in an elderly woman: a case report. J Med Assoc Thai 2012; 95: 723-36.

10. Kumar P, Sharma A. Gonadotropin-releasing hormone analogs: understanding advantages and limitations. J Hum Reprod Sci 2014; $7:$ 170-4.

11. Whirledge S, Cidlowski JA. Glucocorticoids, stress, and fertility. Minerva Endocrinol 2010; 35: 109-25.

12. Liu YT, Shi HH, Yu X, Wang S, Fan QB, Liu HY. [Clinical analysis of pelvic abscess with endometriosis]. Zhonghua Fu Chan Ke Za Zhi 2017; 52: 164-7. Chinese.

13. Gobello C. Dopamine agonists, anti-progestins, anti-androgens, long-term-release $\mathrm{GnRH}$ agonists and anti-estrogens in canine reproduction: a review. Theriogenology 2006; 66: 1560-7.

14. Camisa C, Sexton C, Friedman C. Treatment of hidradenitis suppurativa with combination hypothalamic-pituitary-ovarian and adrenal suppression. A case report. J Reprod Med 1989; 34: 543-6.

15. Malvadkar SM, Malvadkar MS, Domkundwar SV, Mohd S. Spontaneous rupture of pyometra causing peritonitis in elderly female diagnosed on dynamic transvaginal ultrasound. Case Rep Radiol 2016; 2016: 1738521. 